\title{
HIGH TEMPERATURE OXIDATION OF NB-CONTAINING ZR ALLOY CLADDING IN LOCA CONDITIONS
}

\author{
TOSHINORI CHUTO*, FUMIHISA NAGASE and TOYOSHI FUKETA \\ Fuel Safety Research Group, Nuclear Safety Research Center, Japan Atomic Energy Agency \\ 2-4 Shirakata-shirane, Tokai-mura, Naka-gun, Ibaraki 319-1195, Japan \\ "Corresponding author. E-mail : chuto.toshinori@jaea.go.jp
}

Received December 28, 2008

Accepted for Publication January 16, 2009

\begin{abstract}
In order to evaluate high-temperature oxidation behavior of the advanced alloy cladding under LOCA conditions, isothermal oxidation tests in steam were performed with cladding specimens prepared from high burnup PWR fuel rods that were irradiated up to $79 \mathrm{MWd} / \mathrm{kg}$. Cladding materials were $\mathrm{M}^{\circledR}$ and $\mathrm{ZIRLO}{ }^{\mathrm{TM}}$, which are $\mathrm{Nb}$-containing alloys. Ring-shaped specimens were isothermally oxidized in flowing steam at temperatures from 1173 to $1473 \mathrm{~K}$ for the duration between 120 and 4000s. Oxidation rates were evaluated from measured oxide layer thickness and weight gain. A protective effect of the preformed corrosion layer is seen for the shorter time range at the lower temperatures. The influence of pre-hydriding is not significant for the examined range. Alloy composition change generally has small influence on oxidation in the examined temperature range, though $\mathrm{M} 5^{\circledR}$ shows an obviously smaller oxidation constant at $1273 \mathrm{~K}$. Consequently, the oxidation rates of the high burnup $\mathrm{M}^{\circledast}{ }^{\circledast}$ and $\mathrm{ZIRLO}{ }^{\mathrm{TM}}$ cladding are comparable or lower than that of unirradiated Zircaloy-4 cladding.
\end{abstract}

KEYWORDS : LOCA, High Burnup Fuel, Cladding, Oxidation, Corrosion, Hydriding, Reaction Kinetics

\section{INTRODUCTION}

During a postulated loss-of-coolant accident (LOCA) in a light water reactor (LWR), fuel cladding is oxidized due to zirconium-steam reaction at high temperatures. This leads to the growth of brittle layers, such as zirconium dioxide $\left(\mathrm{ZrO}_{2}\right)$ layers and oxygen-stabilized $\alpha-\mathrm{Zr}(\mathrm{O})$ layers, as well as an increase of oxygen concentration in the ductile $\beta-\mathrm{Zr}$ phase. Fuel cladding loses ductility when severely oxidized, and thus the LOCA criteria require that the oxidation shall not exceed a certain limit $(15 \%$ of cladding thickness, in Japan).

As the fuel burnup increases, corrosion layers grow, hydrogen absorption becomes more significant and radiation damage is accumulated in fuel cladding. Since the reaction at the cladding surface and the oxygen diffusion in the layers are the key processes of the oxidation, these high burnup factors may have an influence on the oxidation kinetics. Therefore, effects of those high-burnup factors have been investigated for unirradiated and irradiated Zircaloy cladding, and it was reported that the presence of a pre-corrosion layer may suppress the high-temperature oxidation, while the influence of pre-hydriding is very small [1-4].

The trend toward increased fuel burnup in LWRs is likely to continue. In addition, advanced alloys are being developed to improve corrosion resistance and help achieve higher burnup. Although performance of these alloys has been examined for normal operation conditions, investigations on the cladding behavior of such materials under accident conditions are limited, including for high temperature oxidation.

Since the LOCA criteria define the maximum cladding oxidation as described above, it is necessary to evaluate the oxidation kinetics of the high burnup cladding for the safety analysis. The oxidation kinetics data is also used to evaluate heat generation and hydrogen production during the accidents.

In the present study, isothermal oxidation testing was performed with specimens prepared from high burnup PWR fuel rods with $M 5^{\circledR}$ and $\mathrm{ZIRLO}^{\mathrm{TM}}$ fuel cladding in order to investigate the effects of corrosion and hydrogen absorption during reactor operation on the high temperature oxidation of the advanced alloys.

\section{EXPERIMENTAL PROCEDURES}

\subsection{Sample Preparation}

Table 1 shows a list of the cladding specimens. The specimens were prepared from three PWR fuel rods that had been irradiated to 66,67 or $79 \mathrm{MWd} / \mathrm{kg}$ (local burnup) at European power plants. The cladding materials were M5 (Zr-1.0\% Nb) and ZIRLO (Zr-1.0\% Sn-0.1\% Fe-1.0\% 
Table 1. Specimens used in Oxidation Tests

\begin{tabular}{ccccc}
\hline Specimen ID & Local burnup (MWd $/ \mathrm{kg})$ & Cladding material & Corrosion layer $(\mu \mathrm{m})$ & Hydrogen content $(\mathrm{ppm})$ \\
\hline MFI1-5 & 66 & M5 & 6 & $75-77$ \\
MFI6-15 & 67 & & 7 & $66-68$ \\
\hline ZIR1-13 & 79 & ZIRLO & $84-98$ & $904-988$ \\
\hline
\end{tabular}

Table 2. Conditions of Oxidation Tests

\begin{tabular}{cccc}
\hline \multirow{2}{*}{ Specimen ID } & Material & \multicolumn{2}{c}{ Oxidation conditions } \\
\cline { 3 - 4 } & & Temperature (K) & Time (s) \\
\hline MFI & irradiated M5 & $1273-1473$ & $120-2760$ \\
FA / FH & unirradiated M5 (as-fabricated / pre-hydrided) & $1273-1473$ & $120-3600$ \\
MDA & unirradiated MDA & $1173-1473$ & $120-4000$ \\
ZIR & irradiated ZIRLO & $1173-1473$ & $120-4000$ \\
\hline
\end{tabular}

$\mathrm{Nb}$ ). Corrosion layer thickness and hydrogen concentration were determined by microstructure observation and hydrogen analysis with the hot extraction method for specimens taken from the same fuel rods. Short segments were cut from the mother rods. Fuel pellets were mechanically removed as much as possible by drilling, because the scope of this study extends only to the influence of corrosion and hydrogen absorption, not to the pellet or pellet-cladding bonding layer. Specimens of $10 \mathrm{~mm}$ in length were cut from the resulting segments. Because of strong bonding between pellets and cladding, the cladding specimens of M5 alloy with a very thin layer of fuel pellet periphery were subjected to the oxidation tests. As for the ZIRLO cladding, fuel remnant and pellet-cladding bonding layer on cladding inner surface were completely removed. Therefore, the inner surface of the ZIRLO cladding was in direct contact with the steam, while the M5 cladding had a thin fuel and bonding layer. After cleaning with acetone, dimensions and initial weights of the specimens were measured. Three kinds of unirradiated specimens were also examined; as-fabricated and prehydrided (about $150 \mathrm{ppm}$ ) specimens of M5 alloy cladding, and as-fabricated MDA ( $\mathrm{Zr}-0.8 \% \mathrm{Sn}-0.2 \% \mathrm{Fe}-0.1 \% \mathrm{Cr}-$ $0.5 \% \mathrm{Nb}$ ) cladding.

\subsection{Apparatus and Test Procedure}

Fig. 1 shows a schematic of oxidation test apparatus. The apparatus consists of a ceramic reaction tube, an electrical resistance furnace and a steam generator. Steam

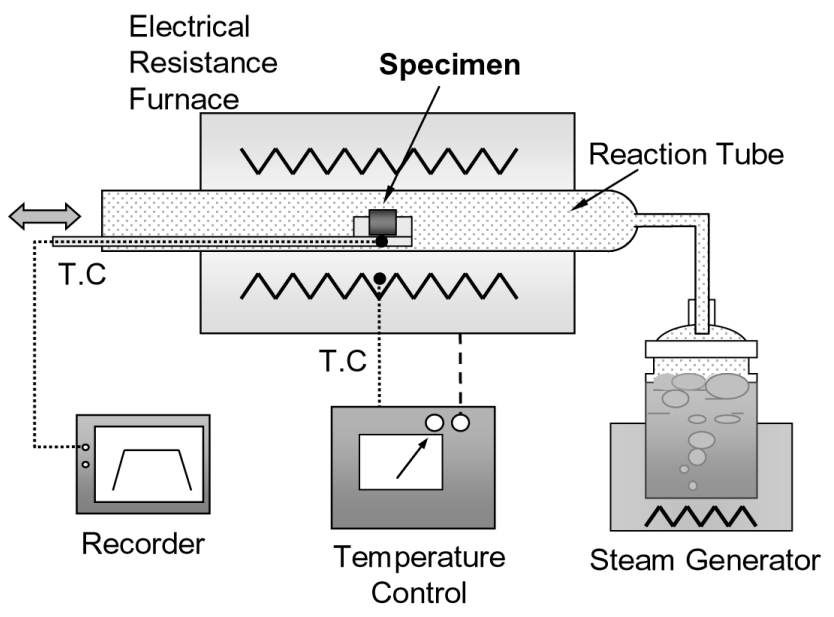

Fig. 1. Schematic of Oxidation Test Apparatus

flow was maintained at a constant rate in the reaction tube during the oxidation test. The furnace and reaction tube were first stabilized at the desired test temperature. The specimens put on a quartz boats were then individually inserted into the midpoint of the tube and the outer and the inner surface of the specimens were oxidized in flowing steam. After their respective pre-determined oxidation duration, the specimens were quickly pulled out of the 


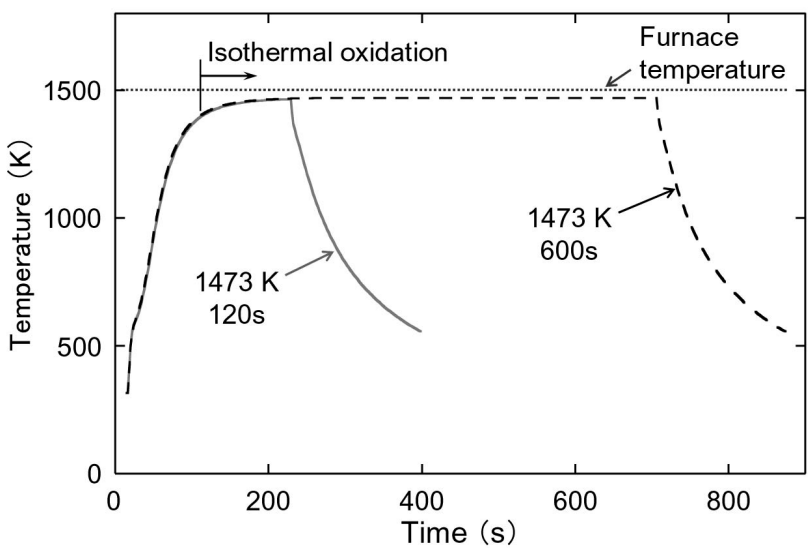

Fig. 2. Example of Temperature Histories

hot zone and cooled. Temperature was measured with an R-type thermocouple at the specimen holder. Specimen temperature was estimated from the holder temperature and the temperature difference between the holder and the specimen, which was measured in the preliminary tests.

Conditions of the oxidation tests are summarized in Table 2. Isothermal oxidation was performed at temperatures from 1173 to $1473 \mathrm{~K}$ for the duration between 120 and 4000s.

Fig. 2 shows typical temperature histories in the tests at $1473 \mathrm{~K}$ with isothermal periods of 120 and 600s. The figure indicates that heating rate and oxidation temperature are the same irrespective of the oxidation time. Since a decrease of the heating rate was observed when specimens approached oxidation temperature, calculation of the isothermal oxidation time was begun when the specimen temperature reached $98 \%$ to the target temperatures. The heat-up periods were about 160, 140, 130, 120 and 100s in the tests at $1173,1273,1323,1373$ and $1473 \mathrm{~K}$, respectively. The temperature variation was within $10 \mathrm{~K}$ for the target temperature during the isothermal period.

After the oxidation test, the weight of each specimen was measured by means of a direct reading balance and the weight gain per unit surface area was calculated. Microstructure observation of the cladding cross section was carried out and thickness of oxide layer was measured.

\section{RESULTS AND DISCUSSION}

\subsection{Oxidation of M5 Cladding}

The weight gains of the unirradiated M5 cladding specimens oxidized at different temperatures are plotted as a function of the square root of oxidation time in Fig. 3. Those of unirradiated (as-fabricated) Zircaloy-4 (Zry-4) cladding [5] are also shown in the figure for comparison.

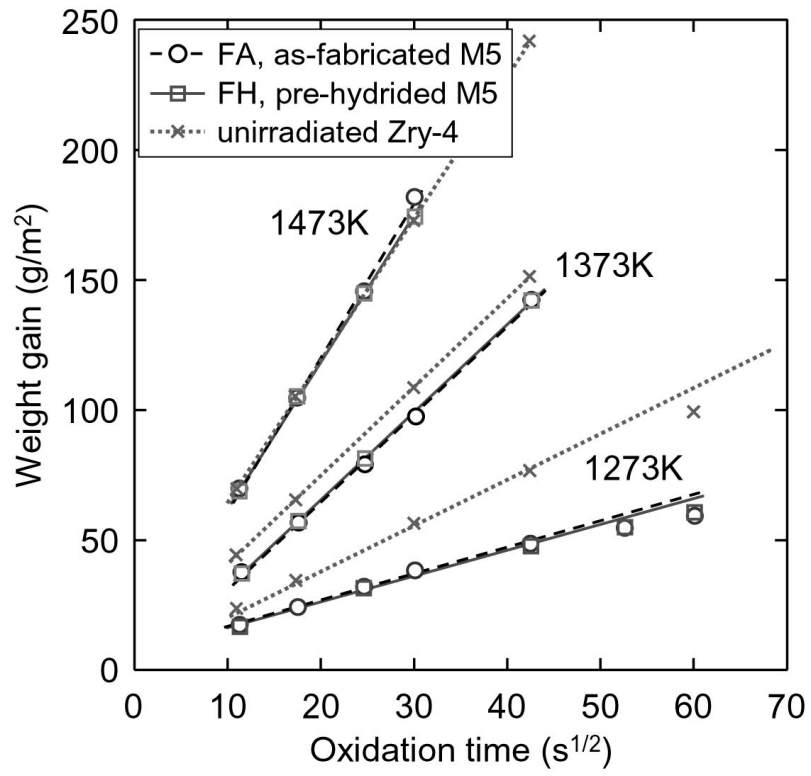

Fig. 3. Weight Gains of Unirradiated M5 and Zry-4 Cladding, Oxidized at 1273, 1373 and $1473 \mathrm{~K}$, as a Function of the Square Root of Oxidation Time

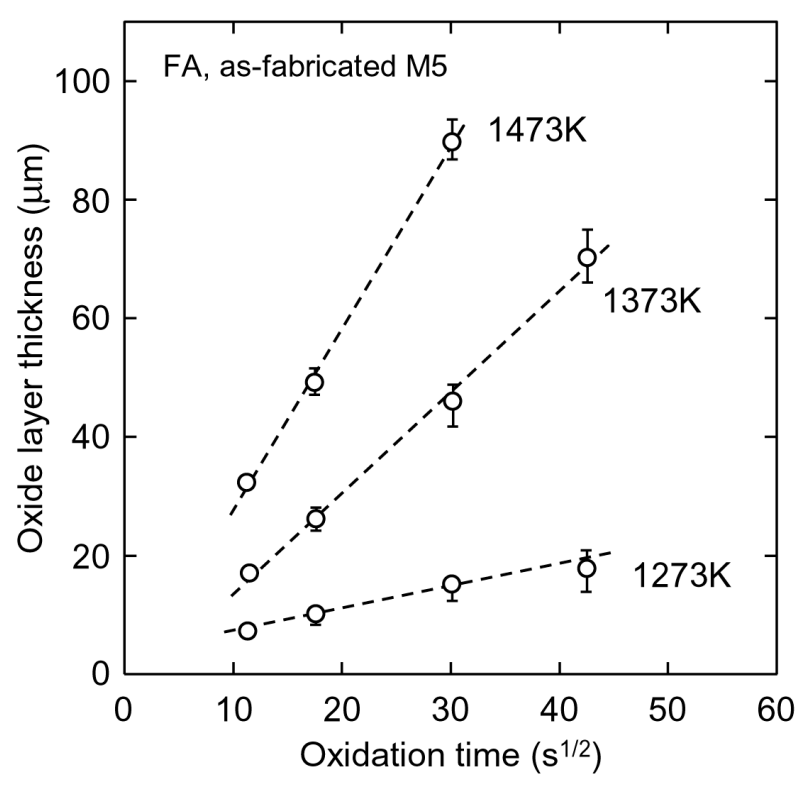

Fig. 4. Oxide Layer Growth in Unirradiated M5 Cladding, Oxidized at 1273,1373 and $1473 \mathrm{~K}$, as a Function of the Square Root of Oxidation Time

A linear relationship is generally found between the weight gain and the square root of oxidation time at every tested 


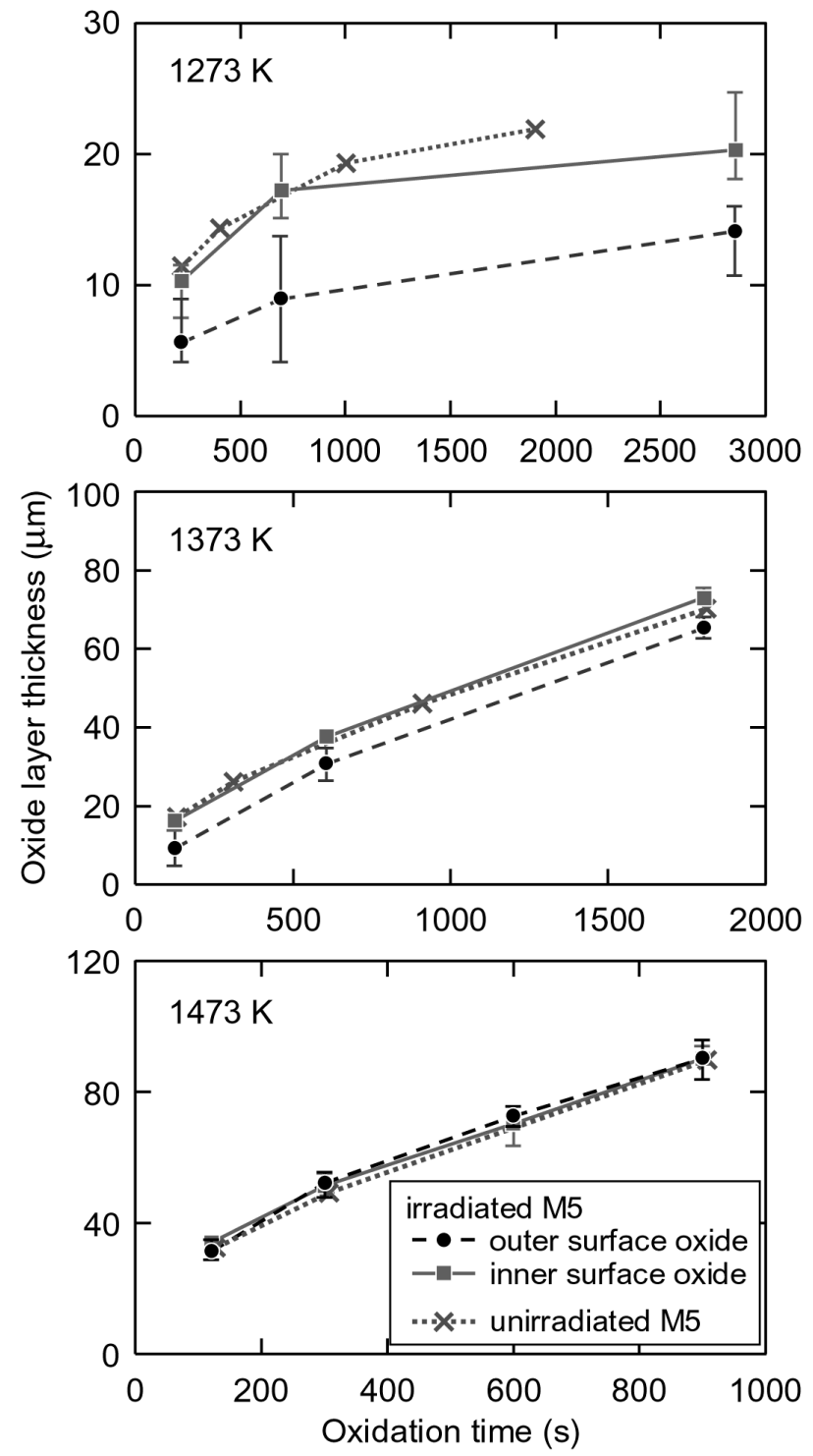

Fig. 5. Oxide Layer Growth in Irradiated and Unirradiated M5 Cladding, Oxidized at 1273, 1373 and $1473 \mathrm{~K}$, as a Function of Oxidation Time

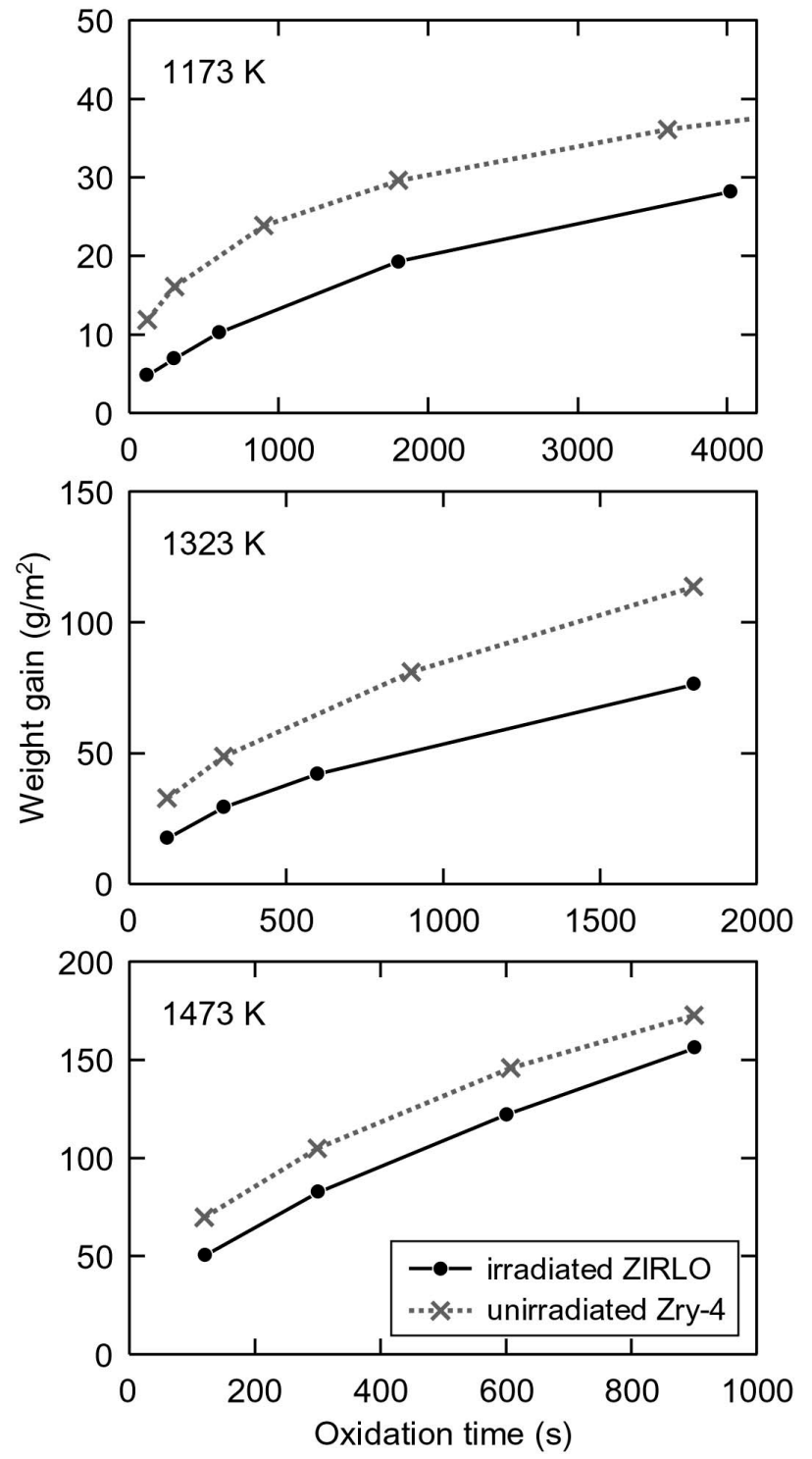

Fig. 6 Weight Gains of Irradiated ZIRLO and Unirradiated Zry-4 Cladding, Oxidized at 1173,1323 and $1473 \mathrm{~K}$, as a Function of Oxidation Time temperature. The correlation between the thickness of the oxide layer and the square root of oxidation time is shown in Fig. 4. The relationship between the thickness of the oxide layer and the square root of the oxidation time is also linear. Therefore, the oxidation of the unirradiated M5 cladding follows parabolic kinetics for the examined range. The oxidation rates derived from the weight gain of the unirradiated M5 cladding are comparable with those of the unirradiated Zry-4 cladding at 1373 and $1473 \mathrm{~K}$, while they are lower at $1273 \mathrm{~K}$. Comparison of the weight gain between as-fabricated and pre-hydrided M5 cladding shows that the effect of pre-hydriding is negligible on the oxidation rates.

Oxide layer thickness measured on the microphotographs of the irradiated and the unirradiated M5 cladding is plotted in Fig. 5 as a function of oxidation time. The error bars indicate the range from the maximum and minimum thickness. Growth of the inner surface oxide in the irradiated cladding is almost equivalent to that in the as-fabricated cladding, while the outer surface oxide is obviously thinner in the specimens oxidized at $1273 \mathrm{~K}$. This confirms the protective effect of the corrosion layer formed during reactor operation. The effect is reduced, however, as oxidation temperature increases. 
(a) ZIR8: $1323 \mathrm{~K}, 600 \mathrm{~s}$

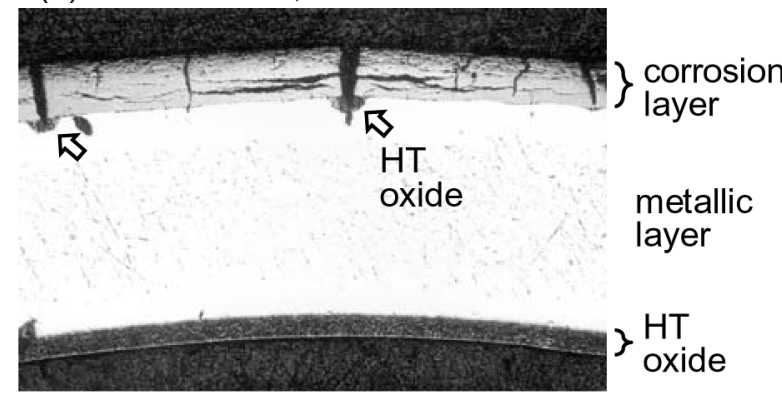

(b) ZIR12: $1473 \mathrm{~K}, 600 \mathrm{~s}$

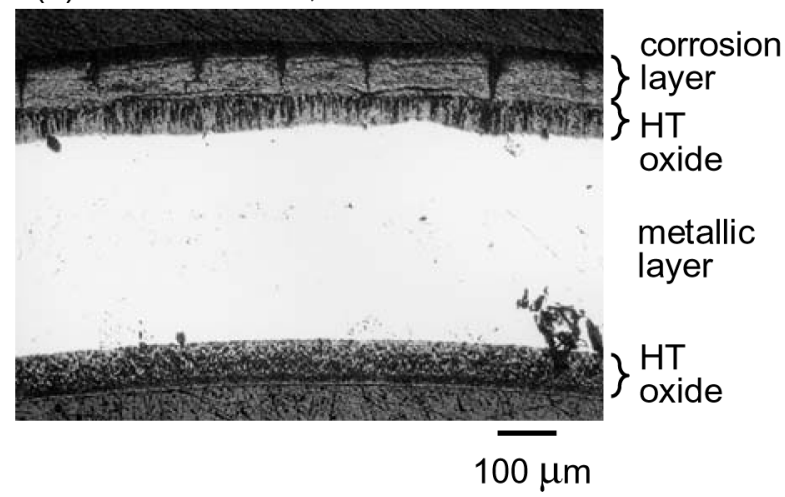

Fig. 7. Cross Sections of Irradiated ZIRLO Cladding after Oxidation Test at (a) $1323 \mathrm{~K}$ and (b) $1473 \mathrm{~K}$ for $600 \mathrm{~s}$

\subsection{Oxidation of ZIRLO Cladding}

The weight gains due to high-temperature oxidation in the irradiated ZIRLO cladding specimens are plotted as a function of oxidation time in Fig. 6. The data for unirradiated Zry- 4 specimens tested in the similar conditions are also shown in the figure for comparison. For all the tested temperatures, the weight gain is smaller in the irradiated ZIRLO specimens for the examined time range. Since it has been reported that the difference in the oxidation rate between the two is unremarkable in the unirradiated condition [6], the oxidation rate is obviously reduced by high burnup. The effect of pre-oxidation and corrosion has been reported for unirradiated and irradiated Zry-4 cladding $[3,7]$ and the present result basically agrees with the previous studies. The difference between the irradiated and the unirradiated specimens becomes smaller as temperatures increase, which also agrees with the previous results.

Fig. 7 shows micro-photographs of the irradiated ZIRLO specimens after the oxidation test at 1323 and $1473 \mathrm{~K}$ for $600 \mathrm{~s}$. In all the examined specimens, the preformed corrosion layer remained on the cladding outer surface and many cracks were observed in the layer. The figures show that the high-temperature (HT) oxidation initiated at cracking positions of the corrosion layer on the cladding outer surface and that, at higher temperature or after longer oxidation times, the oxide layer grows uniformly. On the cladding inner surface, the oxide layer was uniform in all tested conditions. The thickness of the HT oxide measured on the micro-photographs is plotted in Fig. 8 as a function of oxidation time. The outer surface oxide is thinner in the irradiated samples in the examined temperature and time range, which is possibly caused by the protective effect of the pre-formed corrosion layer, as observed in Fig. 7. This effect becomes smaller as oxidation temperature increases. The suppression of oxide growth is remarkable in the ZIRLO cladding compared to the
M5 because of the thicker corrosion layer on the ZIRLO cladding. On the other hand, growth of the inner surface oxide in the irradiated ZIRLO cladding is similar to that in the unirradiated Zry-4. Hence, the influence of hydrogen absorption on oxide layer growth is small.

\subsection{Oxidation Kinetics of the Advanced Alloy Cladding}

It is generally accepted that the mechanism which governs the high-temperature oxidation of Zircaloy cladding is the diffusion of oxygen anions through the $\mathrm{ZrO}_{2}$ lattice. The oxidation rate is described by a parabolic expression of the form

$$
\Delta \mathrm{W}^{2}=\mathrm{K}_{\mathrm{W}} \cdot \mathrm{t}
$$

where $\Delta \mathrm{W}$ is the weight gain per unit surface area, $\mathrm{t}$ is the oxidation time, and $\mathrm{K}_{\mathrm{w}}$ is the parabolic rate constant for weight gain. The linear relationship between the measured weight gain and the square root of oxidation time (Fig. 3) shows that high-temperature oxidation of the unirradiated M5 cladding obeys a parabolic rate law indicating a diffusion controlled reaction. The temperature dependence of the obtained parabolic rate constants for the unirradiated M5 cladding is shown in Fig. 9 together with the data for the unirradiated Zry-4 cladding [5]. The figure indicates that the rate constants of the M5 and Zry4 alloys are equivalent at 1373 and $1473 \mathrm{~K}$, and that that of the M5 alloy is lower at $1273 \mathrm{~K}$. This agrees with the results by Portier et al. [4]. Since the unirradiated MDA cladding showed a linear relationship between the measured weight gain and the square root of oxidation time, the rate constants were evaluated and plotted in the figure. The rate constants of the unirradiated MDA are equivalent to that of the unirradiated Zry-4 for the examined range. 

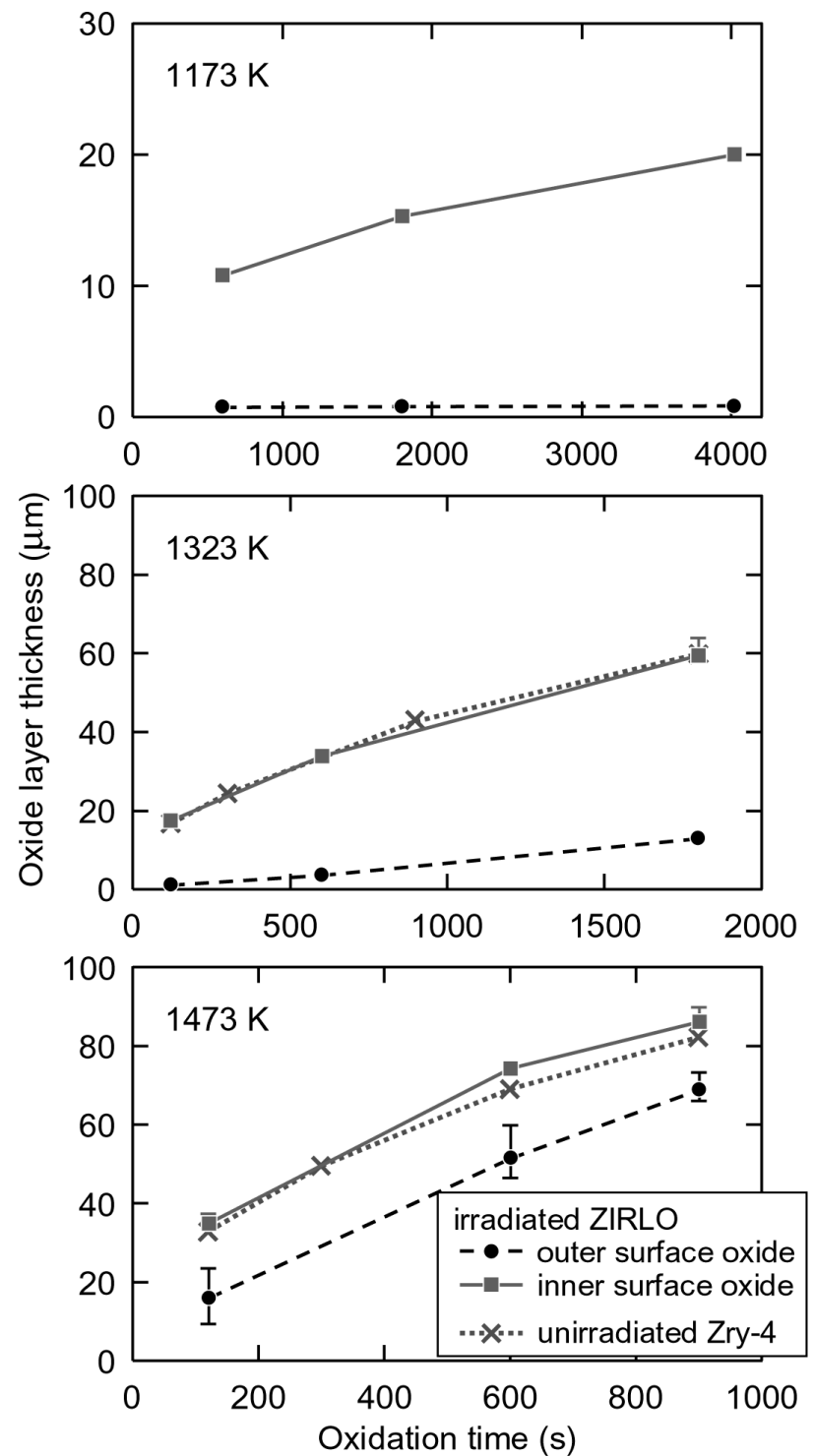

Fig. 8. Oxide Layer Growth in Irradiated ZIRLO and Unirradiated Zry-4 Cladding, Oxidized at 1173, 1323 and $1473 \mathrm{~K}$, as a Function of Oxidation Time

It has been reported that the unirradiated ZIRLO cladding exhibited comparable oxidation rates [6]. Consequently, the influence of the composition changes including $\mathrm{Nb}$ addition in the advanced alloys is generally small on the oxidation kinetics at temperatures higher than $1273 \mathrm{~K}$, though the M5cladding has a smaller oxidation constant at $1273 \mathrm{~K}$.

Assuming that the oxidation rate of the irradiated cladding also follows parabolic kinetics, the parabolic rate constants were evaluated by a linear regression method using the method of the least squares, and the rate constants of the irradiated ZIRLO cladding are plotted in Fig. 9.

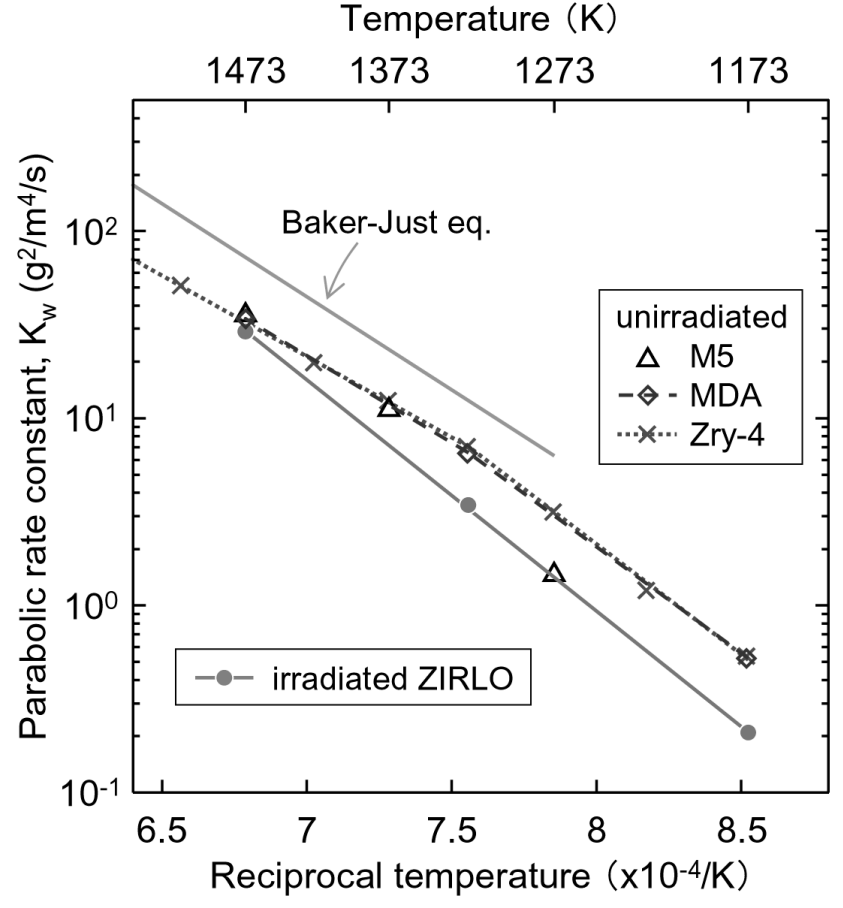

Fig. 9. Temperature Dependence of the Parabolic Rate Constants for Weight Gain

The rate constants are lower than those of the unirradiated cladding at $1373 \mathrm{~K}$ and below, while difference is small at $1473 \mathrm{~K}$. The equation describing the temperature dependence of the parabolic rate constants of the irradiated ZIRLO cladding is

$$
\mathrm{K}_{\mathrm{W}}=5.75 \times 10^{9} \exp \left(-2.33 \times 10^{5} / \mathrm{RT}\right)
$$

The unit of the parabolic rate constant, $\mathrm{K}_{\mathrm{w}}$, is $\mathrm{g}^{2} / \mathrm{m}^{4} / \mathrm{s}$, the universal gas constant $\mathrm{R}$ is $8.314 \mathrm{~J} / \mathrm{mol} / \mathrm{K}$ and $\mathrm{T}$ is the absolute temperature in $\mathrm{K}$. The weight gains measured in the irradiated M5 cladding were obviously lower than those in the unirradiated M5 cladding at every tested temperature. However, the rate constant of the irradiated cladding could not be evaluated because of large deviation from the linear relationship. The specimens were subjected to the oxidation test with a thin layer of the fuel pellets. Hence, the large deviation may be caused by drop of pellet fragments during the test.

Systematic study was performed at Japan Atomic Energy Agency (JAEA) on the influence of pre-hydriding on oxidation rate and it was shown that the hydrogen effect produces less than a $5 \%$ difference in the oxidation rate under realistic conditions ( $<800 \mathrm{ppmH}$ and $<1800 \mathrm{~s}$ ) [3]. As mentioned above, the oxidation rate of the unirradiated M5 cladding little changed by pre-hydriding up to 150 
ppm, and there is no obvious effect of absorbed hydrogen on the growth of the oxide layer on the inner surface of the irradiated ZIRLO and M5 cladding. Accordingly, the influence of hydrogen on high temperature oxidation of the advanced alloy cladding would be small as well as Zircaloy-4 cladding.

As described, the oxidation rate at high temperatures is reduced by the existence of the corrosion layer formed during reactor operation, though it is limited for the shorter time range and the lower temperature range. Microstructure observation shows that high temperature oxidation initiates at cracks in the corrosion layer, and that the local oxidation grows to form a uniform oxide layer beneath the corrosion layer. It is considered that the corrosion layer has a protective effect against the high temperature oxidation and that this protective effect is gradually reduced by the oxide layer growth which initiates from cracks in the corrosion layer. Microstructure observation after "integral thermal shock tests," simulating the whole LOCA sequence, shows that fine and dense cracks are generated in the corrosion layer of ballooned and ruptured cladding [8]. In such a case, it is not expected that the corrosion layer would provide the same extent of protective effect, and the oxidation rate is considered to become equivalent to that of the unirradiated cladding.

"Breakaway oxidation" is an important phenomenon to consider during a safety evaluation because the oxidation rate increases after the occurrence of breakaway oxidation, and because breakaway oxidation is accompanied by significant hydrogen absorption. It is reported that breakaway oxidation occurs between 773 and $1073 \mathrm{~K}$ and at about $1273 \mathrm{~K}$ for longer than 3600 s in unirradiated Zircaloy [5]. No evidence of breakaway oxidation was seen in any of the specimens tested in the present study. Therefore, it is not necessary to take account of breakaway oxidation for the tested high burnup cladding in the examined temperature and time ranges.

The temperature dependence of the rate constant calculated by the Baker-Just equation [9] used in the safety analysis is also shown in Fig. 9. The figure indicates that the Baker-Just equation remains conservative for the high burnup fuel cladding, providing a sufficient margin.

\section{CONCLUSIONS}

In order to investigate the influence of high burnup on the high-temperature oxidation of advanced alloy cladding, oxidation tests were performed at temperatures from 1173 to $1473 \mathrm{~K}$ for times between 120 and 4000 s in steam flow. The specimens were sampled from high burnup PWR fuel rods $(<79 \mathrm{MWd} / \mathrm{kg}$ ) with M5 and ZIRLO cladding. Oxidation rates were evaluated from measured oxide layer thickness and weight gain.

High temperature oxidation of the advanced alloy cladding obeyed a parabolic rate law in the examined temperature and time range. Influence of hydrogen absorption on the oxidation kinetics is small for the examined range, while presence of a pre-formed corrosion layer may have a protective effect. Changes in the alloy composition, including $\mathrm{Nb}$ addition, in the advanced alloys generally show little influence on the oxidation kinetics in the examined temperature range, though the M5 cladding has smaller oxidation constant at $1273 \mathrm{~K}$. Consequently, the oxidation rates of the high burnup M5 and ZIRLO cladding are comparable or lower than that of unirradiated Zircaloy-4 cladding. The Baker-Just oxidation kinetics equation remains conservative in relation to the safety analysis for the high burnup fuel cladding in a LOCA, providing a sufficient margin.

\section{ACKNOWLEDGMENTS}

The present study was conducted as a part of program sponsored and organized by Nuclear and Industrial Safety Agency, Ministry of Economy, Trade and Industry. The authors would like to acknowledge and express their appreciation for the time and effort devoted by numerous engineers and technicians in Department of Hot Laboratories and Facilities, JAEA.

\section{REFERENCES}

[1] M. Ozawa, T. Takahashi, T. Homma, and K. Goto, "Behavior of irradiated Zircaloy-4 fuel cladding under simulated LOCA conditions," Zirconium in the Nuclear Industry: $12^{\text {th }}$ International Symposium, ASTM STP 1354, West Conshohocken, Pennsylvania, USA, p. 279 (2000).

[2 ] J. -C. Brachet, V. Maillot, L. Portier, D. Gilbon, A. Lesbros, N. Weackel, and J. -P. Mardon, "Hydrogen content, preoxidation, and cooling scenario effects on post-quench microstructure and mechanical properties of Zircaloy-4 and M5 alloys in LOCA conditions," Zirconium in the Nuclear Industry: 15 $5^{\text {th }}$ International Symposium, Sun River, Oregon, USA, February 24-28, 2007.

[3 ] F. Nagase, T. Otomo, H. Uetsuka, "Experiments on high burnup fuel behavior under LOCA conditions at JAERI," 2000 International Topical Meeting on Light Water Reactor Fuel Performance, Park City, Utah, USA, April 10-13, 2000.

[ 4 ] L. Portier, T. Bredel, J. -C. Brachet, V. Maillot, J. -P. Mardon, and A. Lesbros, "Influence of long service exposures on the thermal-mechanical behavior of $\mathrm{Zy}-4$ and $\mathrm{M} 5^{\mathrm{TM}}$ alloys in LOCA conditions," Zirconium in the Nuclear Industry: 14th International Symposium, Stockholm, Sweden, January 13-15, 2004.

[ 5 ] F. Nagase, T. Otomo and H. Uetsuka, "Oxidation Kinetics of Low-Sn Zircaloy-4 at the Temperature Range from 773 to 1573 K," J. Nucl. Sci. Technol., 40, 4, p.213 (2003).

[6] Y. Yan, T. Burtseva and M. Billone, "LOCA-Related Research at ANL," Proc. of Fuel Safety Research Meeting 2004, Tokyo, Japan, March 1-2, 2004.

[7] F. Nagase, "High burnup fuel behavior under LOCA conditions," Proc. Fuel Safety Research Meeting 2005, Tokyo, Japan, March 2-3, 2005.

[ 8 ] F. Nagase, T. Chuto, T. Fuketa, "Fracture resistance of high burnup PWR fuel cladding under simulated LOCA 
CHUTO et al., High Temperature Oxidation of Nb-containing Zr Alloy Cladding in LOCA Conditions

conditions," 2008 Water Reactor Fuel Performance Meeting, Seoul, Korea, Oct. 19-23, 2008.

[9] L. Baker and L. C. Just, Studies of Metal-Water Reaction at High Temperatures III. Experimental and Theoretical Studies of the Zirconium-Water Reaction, ANL-6548, Argonne National Laboratory (1962). 